\title{
Condição ocular entre trabalhadores de uma indústria metalúrgica brasileira
}

\section{Ocular condition in a brazilian ironmaster industry}

Thais Zamudio Igami', Silvana Artioli Schellini ${ }^{2}$, Olívia Matai ${ }^{3}$, Carlos Roberto Padovani ${ }^{4}$

\section{ResUMO}

Objetivo: Avaliar a condição ocular em população de trabalhadores de uma indústria metalúrgica paulista. Métodos: Amostra de 2516 funcionários de uma fábrica na cidade de São Paulo foi submetida a uma avaliação oftalmológica como exame ocupacional periódico. Foi aplicado um questionário e realizado o exame de acuidade visual (Snellen) e teste de Ishihara. Os funcionários com acuidade visual menor que 0,7 ou com diferenças maior que duas linhas ou que apresentassem alguma queixa ocular, passaram por avaliação complementar (biomicroscopia, refração, tonometria e fundo de olho). Prescreveu-se óculos conforme indicação. Resultados: Houve predomínio do sexo masculino $(62,5 \%)$ e faixa etária de 20 a 29 anos (41\%). A maioria não apresentava antecedentes oculares $(97,6 \%)$ ou sistêmicos $(96,6 \%)$. A acuidade visual estava acima de 0,7 em $95,5 \%$ dos olhos e $84 \%$ não utilizavam correção. O diagnóstico final foi exame normal em $55 \%$ dos casos, presbiopia em $13.6 \%$, astigmatismo miópico em $10 \%$ e hipermetrópico em 7,7\% dos indivíduos. Baixa visão foi encontrada em 2,4\%, cegueira unilateral em $0,4 \%$, não havendo casos de cegueira ou de deficiência visual entre os trabalhadores. As principais causas de baixa visão foram erros refrativos e ambliopia. Conclusão: A maioria dos funcionários da indústria pesquisada apresentava exame oftalmológico normal e nenhum deles apresentava cegueira bilateral.

Descritores: Saúde ocular; Baixa visão; Erros de refração; Promoção da saúde; Saúde do trabalhador; Serviços de saúde do trabalhador; Questionários; Acuidade visual;Testes visuais; Testes de percepção de cores

\footnotetext{
${ }^{1}$ Residente de oftalmologia da Santa Casa de Misericórdia São Paulo - São Paulo (SP), Brasil;

${ }^{2}$ Livre-docente, professora do departamento de oftalmologia, otorrinolaringologia e Cirurgia de Cabeça e Pescoço da Faculdade de Medicina da Universidade Estadual Paulista "Julio de Mesquita Filho" - UNESP - Botucatu (SP), Brasil;

${ }^{3}$ Estagiária de plástica ocular do departamento de oftalmologia, Otorrinolaringologia e Cirurgia de Cabeça e Pescoço da Faculdade de Medicina da Universidade Estadual Paulista "Julio de Mesquita Filho" - UNESP - Botucatu (SP), Brasil;

"Professor titular do departamento de Bioestatística, Instituto de Biociências da Universidade Estadual Paulista "Julio de Mesquita Filho" - UNESP - Botucatu (SP), Brasil.
}

Recebido para publicação em: 29/7/2008 - Aceito para publicação em 22/10/2008 


\section{INTRODUÇÃO}

A $\mathrm{s}$ causas de cegueira variam de acordo com os grupos populacionais que são investigados. $\mathrm{Na}$ região mais industrializada da Europa, as causas de cegueira englobam degeneração macular senil, catarata, glaucoma, retinopatia diabética e erros refracionais não corrigidos. Já na parte menos desenvolvida, destaca-se catarata, glaucoma e retinopatia diabética ${ }^{(1)}$.

No Brasil, na região Sudeste, por meio de estudo populacional de amostragem domiciliar observou-se como causas de cegueira o erro de refração não corrigido, catarata e doenças retinianas ${ }^{(2)}$.

Levantamentos sobre causas de cegueira e deficiência visual, em geral, são feitos em hospitais, em escolas para cegos, em serviços que prestam assistência oftalmológica gratuita ou em consultórios médicos. Dados sobre causas de cegueira de trabalhadores de indústrias brasileiras não são conhecidos.

Uma vez que no mercado de trabalho há maiores chances de emprego para as pessoas que não possuem deficiência, muito provavelmente o número de cegos que trabalham em indústrias deve ser pequeno. A legislação brasileira impõe a prerrogativa obrigatória de se empregar um percentual de deficientes físicos em certas empresas. Além disso, as indústrias se empenham na promoção de medidas de educação e de tratamento adequadas aos funcionários, visando a prevenção de qualquer tipo de deficiência que possa resultar em problema grave para o trabalhador.

Apesar dos exames oftalmológicos que são feitos periodicamente e de rotina, não há publicações sobre condições oculares em trabalhadores de indústrias. Desta forma, com o objetivo de conhecer a saúde ocular de trabalhadores de uma indústria brasileira, foi realizado este estudo.

\section{Métodos}

O presente estudo teve caráter observacional, transversal, prospectivo, envolvendo 2.516 funcionários de uma indústria metalúrgica da cidade de São Paulo. Uma equipe de triagem (oftalmologistas, residentes, biomédicas e enfermeiras) composta por 12 examinadores foi treinada para aplicação de um questionário contendo os dados de identificação de cada funcionário e perguntas a respeito de queixas oftalmológicas, antecedentes sistêmicos, oculares e familiares.

Em seguida, os funcionários foram avaliados pelo Teste da visão de cores (Ishihara) e Teste de acuidade visual para longe (Tabela de Snellen, com uso de correção óptica quando presente). Estas avaliações foram feitas dentro dos próprios setores da Indústria.

Após a triagem, os indivíduos detectados como portadores de alterações oculares foram submetidos ao exame oftalmológico. Uma Unidade Móvel com suporte para exame oftalmológico completo foi levada até a indústria e os trabalhadores foram avaliados dentro do seu local de trabalho. Os exames foram feitos por 8 oftalmologistas que se revezaram para concluir os atendimentos em 48 horas de serviço ininterrupto.

Os critérios para se indicar o exame oftalmológico completo foram a presença de queixa oftalmológica na abordagem inicial, acuidade visual para longe menor que 0,7 ou a presença de diferença de mais de duas linhas entre os olhos ou presença de queixas oftalmológicas.

O exame oftalmológico completo constou de exame ocular externo, refração, fundoscopia, avaliação da pressão intraocular e exame biomicroscópico. Ao final do exame, quando necessário, foram prescritas lentes corretoras e protetoras, além de orientações gerais e encaminhamento para tratamento especializado, caso estivesse indicado.

Critério de inclusão: todos os trabalhadores da indústria, sem restrições quanto a idade, o sexo ou a condição sócio-econômica.

Critério de exclusão: aqueles que não desejassem participar da pesquisa.

\section{Critérios diagnósticos:}

- Definição de cegueira: Segundo a OMS $(1972)^{(3)}$, a acuidade visual com a melhor correção óptica, no melhor olho, medida para longe, menor que 20/400 (ou 0,05 ). Foi considerado neste estudo também o diagnóstico de cegueira unilateral, seguindo-se o mesmo critério, mas presente em apenas um dos olhos.

- Definição de deficiência visual: A acuidade visual com a melhor correção óptica, no melhor olho, menor que 20/60 $(0,3)$ e maior ou igual do que 20/400 $(0,05)$. Considerou-se também, para este parâmetro, a deficiência visual unilateral e a bilateral.

Avaliação estatística: Os dados obtidos foram transferidos para a planilha Excel. Os resultados estão apresentados quanto à freqüência de ocorrência entre e dentro de populações binomiais.

\section{Resultados}

Foram examinados 2.516 indivíduos que trabalhavam em uma indústria metalúrgica na cidade de São Paulo.

A maior parte da população de trabalhadores apresenta-se entre as faixas etárias de 20 a 29 anos (41\%) 


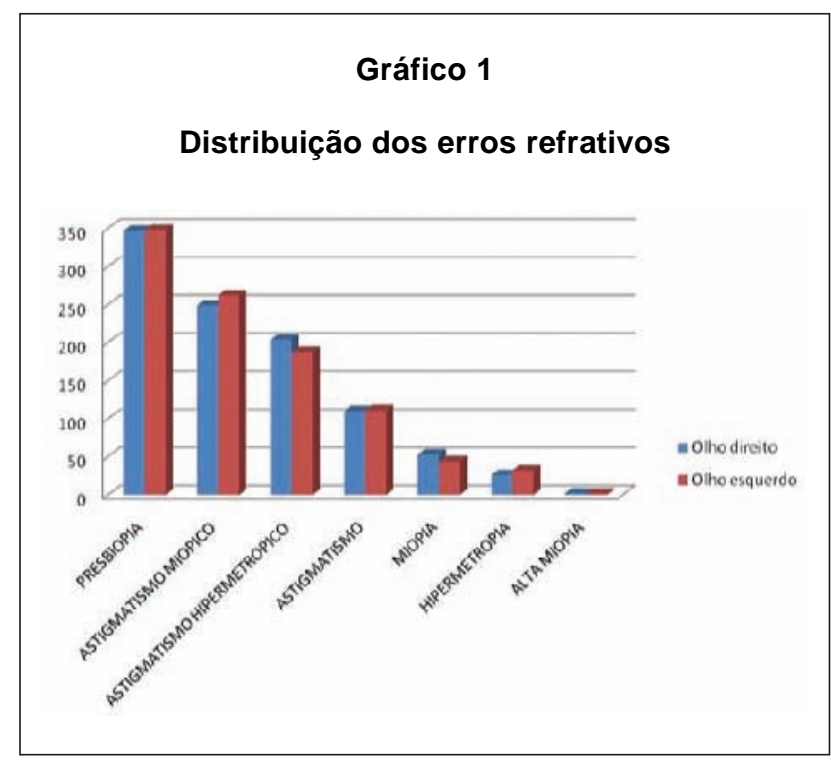

e de 30 a 39 anos (37\%). Houve predomínio do sexo masculino $(62,5 \%)$.

A acuidade visual estava acima de $0,7 \mathrm{em} 95,5 \%$ dos olhos. Considerando-se a presença de erros refrativos, $55 \%$ dos indivíduos foram considerados normais, $13.6 \%$ portadores de presbiopia, $10 \%$ com astigmatismo miópico e $7.7 \%$ com astigmatismo hipermetrópico. Não houve necessidade de prescrição óptica para $84 \%$ do total de trabalhadores.

O Gráfico 1 mostra a distribuição dos diagnósticos refrativos feitos nos indivíduos segundo olho direito e esquerdo. Os erros refrativos mais freqüentes foram a presbiopia seguida pelo astigmatismo composto (miópico ou hipermetrópico). $\mathrm{O}$ astigmatismo simples, assim como a miopia e a hipermetropia foram menos freqüentemente detectados. Vale lembrar que o exame refracional foi feito apenas nos indivíduos que apresentaram acuidade visual menor que 0,7 ou diferença entre os olhos maior que duas linhas, ou queixas oftalmológicas que justificassem a avaliação. Não houve diferença refracional significativa entre os olhos direito e esquerdo.

Deficiência visual unilateral foi detectada em $2,4 \%$ dos trabalhadores desta indústria. Avaliando-se as causas de baixa visão detectadas na indústria, observouse que ambliopia refracional figurou dentre as causas mais freqüentes de cegueira e de deficiência visual nos indivíduos do estudo. O pterígio também foi causa de baixa visão, uma vez que acometia o eixo visual em alguns indivíduos. Trauma antigo, ceratocone, estrabismo, seqüelas retinianas, catarata senil e uveíte também foram detectados, porém em número reduzido de pessoas (Gráfico 2).

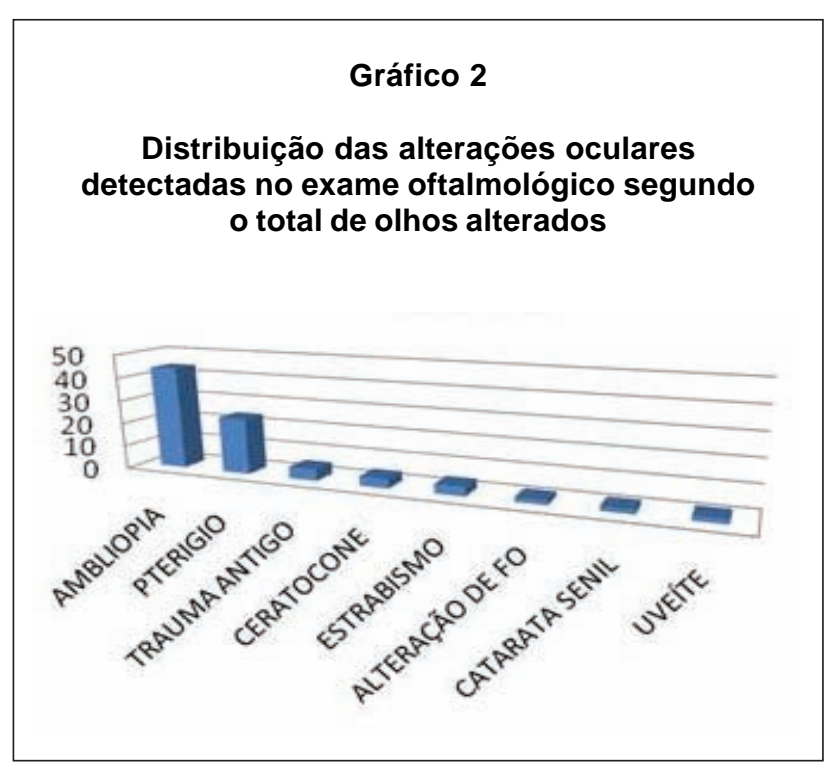

Gráfico 3

Distribuição das condutas adotadas após o exame oftalmológico com relação a prescrição de lentes corretoras para os portadores de alteração visual

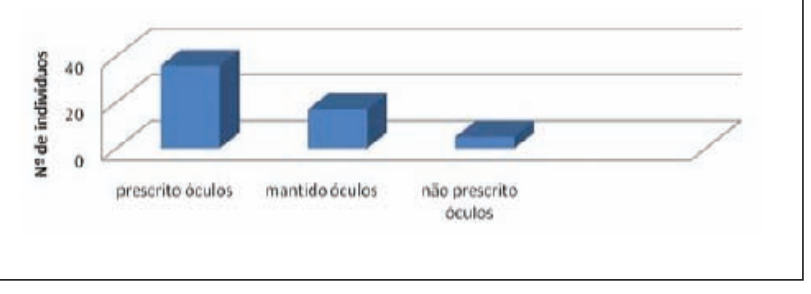

Dos 60 indivíduos (2,4\%) detectados como portadores de deficiência visual, houve necessidade de prescrição de correção óptica, conforme demonstrado no Gráfico 3.

Quanto aos portadores de cegueira, foram detectados 10 olhos cegos unilaterias $(0,4 \%)$. Não houve cegueira bilateral. Observou-se que a visão permaneceu ruim mesmo com a correção óptica e que as causas de cegueira detectadas foram a ambliopia em cinco casos (refracional, anisometrópica, estrábica), o estrabismo em dois casos, seqüela de trauma anterior em um caso ou a presença de cicatriz retiniana em um caso; em um indivíduo não se conseguiu determinar a causa de cegueira (Tabela 1). Seguindo-se a definição da OMS que considera a cegueira como o acometimento de ambos os olhos, considera-se que não havia nenhum caso de cegueira em trabalhadores desta indústria. 
Tabela 1

Distribuição dos portadores de cegueira detectados durante o exame oftalmológico, segundo idade, olho e causa de cegueira

\begin{tabular}{cccccc}
\hline & AVSC & AVSC & AVCC & AVCC & \\
\cline { 2 - 5 } Idade (anos) & OD & OE & OD & OE & Causa de Cegueira \\
\hline \multirow{2}{*}{55} & $<0,05$ & 0 & $<0,05$ & 0,8 & Estrabismo \\
25 & $<0,05$ & 0,2 & $<0,05$ & 1,0 & Ambliopia \\
34 & $<0,05$ & 1,0 & $<0,05$ & 1,0 & Cicatriz retiniana \\
53 & $<0,05$ & 1,0 & $<0,05$ & 1,0 & Ambliopia \\
26 & 1,0 & $<0,05$ & 1,0 & $<0,05$ & Trauma \\
37 & 1,0 & $<0,05$ & 1,0 & $<0,05$ & Ambliopia \\
29 & 0,1 & $<0,05$ & 1,0 & $<0,05$ & Ambliopia \\
66 & $<0,05$ & 1,0 & $<0,05$ & 1,0 & Indeterminada \\
42 & $<0,05$ & 1,0 & $<0,05$ & 1,0 & Estrabismo \\
31 & $<0,05$ & 1,0 & $<0,05$ & 1,0 & Ambliopia \\
\hline
\end{tabular}

\section{DiscuSSÃO}

O presente estudo pesquisou causas de cegueira e deficiência visual na população de trabalhadores de uma indústria metalúrgica da cidade de São Paulo, Brasil.

As avaliações de saúde ocular de trabalhadores da indústria são feitas no exame de admissão e nos exames periódicos. Sua importância relaciona-se ao fato de que muitos trabalham em cargos que exigem boa acuidade visual para avaliar a qualidade das peças manufaturadas e para protegerem-se de acidentes que possam ocorrer na manipulação de máquinas capazes de provocar deficiência definitiva de membros.

No tocante a logística utilizada para o atendimento oftalmológico, é importante destacar a necessidade de conhecer a indústria e seu funcionamento, a fim de elaborar uma programação adequada das atividades para toda a equipe.

Deste modo, deve-se chamar a atenção para a metodologia utilizada para atingir a população alvo. Foi elaborada uma programação com intuito de que todos os trabalhadores pudessem ser abordados dentro do seu ambiente de trabalho e durante o expediente. Desta forma, o tempo útil perdido pelo trabalhador foi muito pequeno, não havendo necessidade de paralisar a produção em nenhum dos setores para a realização dos exames. Esta abordagem permitiu que a indústria economizasse gastos e que não fossem descontadas horas de serviço ou de lazer. Houve também um resultado satisfatório para a equipe que realizou os exames, sendo possível abordar um grande número de pessoas em curto espaço de tempo.
A maioria dos trabalhadores possuía idade entre 20 e 30 anos e era do sexo masculino. Este fato condiz com o mercado de trabalho dentro do ramo metalúrgico, que dá maiores oportunidades para homens jovens, embora esteja aumentando a participação de mulheres nos últimos anos. A faixa etária dos indivíduos diminui a chance de se detectar problemas oculares, como foi observado neste estudo.

Apesar de serem preconizadas avaliações oftalmológicas periódicas nas indústrias que possuem certificado de adequação às normas relacionadas à saúde do trabalhador, os resultados das mesmas não são divulgados. A literatura sobre a saúde ocular de trabalhadores da indústria está voltada para a apresentação de estudos que versam sobre indenizações e acidentes de trabalho, ${ }^{(4)}$ sem a preocupação de relatar quais seriam as alterações oculares que acometem o trabalhador, e se estas seriam passíveis de tratamento.

A alteração visual mais freqüente na população estudada foi o erro de refração. Destaca-se a presbiopia, para a qual se faz necessário o atendimento oftalmológico para $100 \%$ das pessoas que se encotram na faixa etária acima dos 40 anos, tendo em vista a perda da acomodação, que prejudica a visão para tarefas à curta distância. Esta situação repete-se em outros locais do mundo, como mostrou um estudo feito na Alemanha, no qual praticamente $100 \%$ dos indivíduos acima dos 55 anos possuía algum grau de alteração visual que requeria correção óptica ${ }^{(5)}$.

Os erros refracionais foram os responsáveis pela maioria dos casos de deficiência visual, encontrando-se casos de altos graus que provocam grandes déficits visuais. Porém, avaliando-se os portadores das ametropias 
após a correção óptica, observaram-se muitos casos que se beneficiaram desta, restando poucos casos com deficiência visual importante ou cegueira.

Entre os indivíduos com erros refracionais, a maioria apresentava astigmatismo composto. Apesar de ser um grupo restrito de pessoas que trabalha dentro de uma indústria, o destaque para o astigmatismo também surge em outro estudo brasileiro ${ }^{(6)}$. Entretanto, para a população japonesa, a miopia é sabidamente o erro refrativo mais importante ${ }^{(7)}$.

A catarata e os erros de refração continuam a ser causas importantes de cegueira reversível, tanto no Brasil $^{(2)}$, como em locais como a Índia e o Paraguai ${ }^{(8-9)}$, mas não em Israel, onde a degeneração macular senil e o glaucoma (14\%), seguidas da retinopatia diabética (11\%), catarata e maculopatia miópica (10\%) e atrofia óptica $(8.4 \%)$ são as principais causas ${ }^{(10)}$. Estudo também transversal, feito na população rural da Índia, mostrou prevalência de cegueira por trauma em $0,8 \%$ dos indivíduos acima de 40 anos, sendo o trauma mais prevalente que o glaucoma, degeneração macular ou retinopatia diabética ${ }^{(11)}$.

A definição de cegueira adotada pela $\mathrm{OMS}^{(3)}$ reza que o melhor olho deva ter acuidade menor ou igual a 0,05 . Os índices de cegueira podem ser estimados para um ou para os dois olhos, embora para efeitos legais, a cegueira unilateral não seja importante. Porém, para avaliação de causas de cegueira na população e para a adoção de medidas preventivas em termos populacionais, justifica-se o estudo de causas de cegueira unilateral. Destaca-se que a ambliopia, doença facilmente prevenível se detectada a tempo,foi responsável por $50 \%$ dos olhos cegos. E o estrabismo, outra causa de cegueira que poderia ter sido evitada, respondeu por outros $20 \%$. Desta forma, o levantamento mostra que as causas de cegueira existentes seriam reversíveis, caso fossem detectadas precocemente, o que possibilitaria o tratamento adequado.

A estimativa de cegueira bilateral feita pelo presente estudo foi de $0 \%$ e de unilateral, de $0,4 \%$. Índices baixos são justificados pelo tipo de atividade exercida pelos trabalhadores.

\section{Conclusão}

A avaliação dos trabalhadores de uma indústria metalúrgica da cidade de São Paulo mostrou 2,4\% de olhos com baixa visão unilateral e $0,4 \%$ com cegueira unilateral, não havendo nenhum caso de cegueira bilateral entre os trabalhadores. Os erros refrativos foram considerados os responsáveis pela maior parte dos casos de deficiência visual. Os autores enfatizam que as características da população (jovem) e os critérios de seleção para ingresso contribuíram para a baixa ocorrência de deficiência visual e cegueira detectados. A importância da realização de exames periódicos freqüentes está na prevenção e na detecção precoce de doenças oculares que possam causar baixa visão permanente no futuro e assim, prejudicar o funcionário nas suas tarefas, com o risco de exclusão do mercado de trabalho.

\section{Abstract}

\section{Purpose: To evaluate the ocular condition in a brazilian} ironmaster industry. Method: A prospective study was conducted in 2516 employees from an ironmaster brazilian industry in São Paulo city, Brazil. A questionnaire was applied and visual acuity and Ishihara test were performed in every individual. A complete ophthalmic evaluation (slit lamp examination, fundoscopy, tonometry and refractometry) was done when the visual acuity was worse than 0.7 or when the worker had a greater diference between the eyes (more than two lines) or when there were ocular complains. Glasses were prescribed when necessary. Results: Most of the evaluated employees in this industry was male $(62.5 \%)$, ageing from 20 to 29 year old (41\%). Visual acuity was normal in 95.5 $\%$ of the eyes. The majority of the individuals (84\%) did not need glasses prescription. Fifty- five percent of the employees were considered as normal eye carriers, $13.6 \%$ presented presbyopia, 10\% presented myopic astigmatism and $7.7 \%$ hyperopic astigmatism. Visual impairment was present in $2,4 \%$ and blindness in one eye of $0,4 \%$ of the studied individuals. The main cause of visual impairment was refractive errors and amblyopia. Conclusion: The majority of the employees, in this particular industry, presented with a normal ophthalmic examination. None of them had bilateral blindness.

Keywords: Eye health; Low vision; Refractive errors; Health promotion; Occupational health; Occupational health services; Questionnaires; Visual acuity; Vision tests; Color perception tests

\section{REFERÊNCIAS}

1. Kocur I, Resnikoff S. Visual impairment and blindness in Europe and their prevention. $\mathrm{Br} \mathrm{J}$ Ophthalmol. 2002;86(7):716-22.

2. Schellini SA, Hoyama E, Cordeiro R, Padovani CR. The prevalence of low vision and blindness in a randon brazilian sample [abstract]. In: ARVO - Annual Meeting; 2006, Fort Lauderdale (FL); Abr 30- Maio 4. v.1. p. 47. 
3. World Health Organization. Change the definition of blindness [Internet]. Geneva, Switzerland: WHO; [cited 2008 Aug 12] Available from: http://www.who.int/blindness/ Change \%20the \%20Definition \%20of\%20Blindness

4. Lipscomb HJ, Dement JM, McDougall V, Kalat J. Work-related eye injuries among union carpenters. Appl Occup Environ Hyg. 1999;14(10):665-76.

5. Stang A, Jöckel KH. Visual disturbances in a populationbased survey of 6962 subjects: the German National Health Examination Survey 1998. Eur J Public Health. 2003;13(3):202-9.

6. Schellini SA, Padovani CR, Padovani CRP. Population-based assessment of refractive error in a brazilian random sample [abstract]. In: ARVO - Annual Meeting; 2006, Fort Lauderdale (FL); Abr 30- Maio 4. v.1. p. 47.

7. Iwano M, Nomura H, Ando F, Niino N, Miyake Y, Shimokata $\mathrm{H}$. Visual acuity in a community-dwelling Japanese population and factors associated with visual impairment. Jpn J Ophthalmol. 2004;48(1):37-43.

8. Thulasiraj RD, Rahamathulla R, Saraswati A, Selvaraj S, Ellwein LB. The Sivaganga eye survey: I. Blindness and cataract. Ophthalmic Epidemiol. 2002; 9(5): 299-312.
9. Duerksen R, Limburg H, Carron JE, Foster A. Cataract blindness in Paraguay-results of a national survey. Ophthalmic Epidemiol. 2003;10(5):349-57.

10. Farber MD. National Registry for the Blind in Israel: estimation of prevalence and incidence rates and causes of blindness. Ophthalmic Epidemiol. 2003;10(4):267-77.

11. Nirmalan PK, Katz J, Robin AL, Tielsch JM, Namperumalsamy $\mathrm{P}, \mathrm{Kim} \mathrm{R}$, et al. Prevalence of vitreoretinal disorders in a rural population of southern India: the Aravind Comprehensive Eye Study. Arch Ophthalmol. 2004;122(4):581-6.

\section{ENDEREÇO PARA CORRESPONDÊNCIA:}

Universidade Estadual Paulista Júlio de Mesquita Filho,

Faculdade de Medicina de Botucatu,

Departamento de Oftalmologia e Otorrinolaringologia

Universidade Estadual Paulista - Campus de Botucatu -

Faculdade de Medicina - UNESP(Depto.OFT/ORL/CCP)

Rubião Junior

CEP 18618-970 - Botucatu - SP - Brasil

email:sartioli@fmb.unesp.br 